\title{
Discovery of the neutron star nature of SLX 1737-282
}

\author{
J. J. M. in 't Zand ${ }^{1,2}$, F. Verbunt ${ }^{1}$, E. Kuulkers ${ }^{1,2}$, C. B. Markwardt ${ }^{3,4}$, A. Bazzano ${ }^{5}$, M. Cocchi ${ }^{5}$, \\ R. Cornelisse ${ }^{1,2}$, J. Heise ${ }^{2}$, L. Natalucci ${ }^{5}$, and P. Ubertini ${ }^{5}$ \\ 1 Astronomical Institute, Utrecht University, PO Box 80000, 3508 TA Utrecht, The Netherlands \\ 2 SRON National Institute for Space Research, Sorbonnelaan 2, 3584 CA Utrecht, The Netherlands \\ 3 Dept. of Astronomy, University of Maryland, College Park, MD 20742, USA \\ 4 NASA Goddard Space Flight Center, Code 662, Greenbelt, MD 20771, USA \\ 5 Istituto di Astrofisica Spaziale e Fysica Cosmica (CNR), Area Ricerca Roma Tor Vergata, \\ Via del Fosso del Cavaliere, 00133 Roma, Italy
}

Received 1 April 2002 / Accepted 23 April 2002

\begin{abstract}
SLX $1737-282$ is a persistent moderately bright X-ray source 1.2 from the Galactic center. X-ray observations with the Wide Field Cameras on BeppoSAX have for the first time revealed the true nature of SLX 1737-282: a 15 min long thermonuclear flash was detected exposing the source as a neutron star in a binary system. The flash was Eddington-limited constraining the distance to between 5 and $8 \mathrm{kpc}$. We analyze BeppoSAX, ROSAT, and RXTE data on SLX 1737-282. The persistent $0.5-200 \mathrm{keV}$ luminosity is close to or less than $1 \%$ of the Eddington limit which implies a rarely-seen circumstance for thermonuclear flash activity.
\end{abstract}

Key words. accretion, accretion disks - binaries: close - X-rays: stars: individual: SLX 1737-282, 2E 1737.5-2817, 1RXS J174043.1-281806, RX J1740.7-2818, AX J1740.7-2818

\section{Introduction}

SLX 1737-282 was discovered with the Spacelab-2/X-Ray Telescope (SL2-XRT) which flew from 29 July to 6 August 1985 (Skinner et al. 1987). SL2-XRT targeted the Galactic center a number of times for a total of $7 \mathrm{hrs}$. SLX 1737-282 was detected with an average flux of $7.7 \times$ $10^{-11} \mathrm{erg} \mathrm{cm}^{-2} \mathrm{~s}^{-1}$ in 3 to $30 \mathrm{keV}$. No timing or spectral features were noticed. Skinner et al. also went back to data from the Einstein galactic plane survey (Hertz \& Grindlay 1983) and found that SLX 1737-282 was clearly present. The Einstein source was later given the name of $2 \mathrm{E} 1737.5$-2817 and provided a $1^{\prime}$-accurate position.

Sakano et al. (2002) reported on all $A S C A$-GIS observations of a $5^{\circ} \times 5^{\circ}$ field centered on the galactic center. The position of SLX 1737-282 (also designated AX J1740.7-2818) was covered on 3 occasions in 1996, 1998, and 1999 with a total exposure time of $61.5 \mathrm{ksec}$ and was detected every time with $0.7-10 \mathrm{keV}$ fluxes between 4.8 and $6.5 \times 10^{-11} \mathrm{erg} \mathrm{cm}^{-2} \mathrm{~s}^{-1}$. The spectrum is consistent with a constant absorbed power law with a photon index $\Gamma$ of $2.17 \pm 0.07$ and $N_{\mathrm{H}}=(2.0 \pm 0.1) \times$ $10^{22} \mathrm{~cm}^{-2}$. The flux varied up to $100 \%$ on time scales of $10^{3}$ to $10^{4} \mathrm{~s}$.

Because of being so close to the Galactic center, SLX 1737-282 was serendipitously targeted in a number of X-ray wide-field monitoring programs such as those carried out with Kvant/COMIS-TTM (In 't Zand 1992;
Emelyanov et al. 2000) and Granat/ART-P (Pavlinsky et al. 1994). The source was never detected in these programs. The upper limits are consistent with the ASCA and SL2-XRT detections.

Despite that a neutron star hypothesis was suggested by the spectrum, variability and flux, definite evidence was lacking; an AGN hypothesis could not be ruled out (e.g., Sakano et al. 2002). This has now changed. We present in this paper the first detection of an X-ray burst from SLX 1737-282 which settles the nature of SLX 1737-282. In addition we discuss ROSAT and RXTE data.

\section{BeppoSAX-WFC observations}

The Wide Field Cameras (WFCs; Jager et al. 1997) on the BeppoSAX satellite (Boella et al. 1997a) carry out a monitoring program in 2 to $28 \mathrm{keV}$ of the galactic center since mid 1996. SLX $1737-282$ is well inside the $40^{\circ} \times 40^{\circ}$ field of view and, through the $5^{\prime}$ angular resolution, well-resolved from nearby sources. The monitoring program till early 2002 totalled a net exposure of $\approx 70$ days. SLX $1737-282$ was never detected in individual observations above an upper limit of roughly $2 \times 10^{-10} \mathrm{erg} \mathrm{cm}^{-2} \mathrm{~s}^{-1}(2-10 \mathrm{keV})$ except for a single 15 min period on 27 March 2000 when a flare was detected from a location consistent with the Einstein position of SLX 1737-282 (see Fig. 1). Figure 2 (top panel) shows the time history of the intensity. 


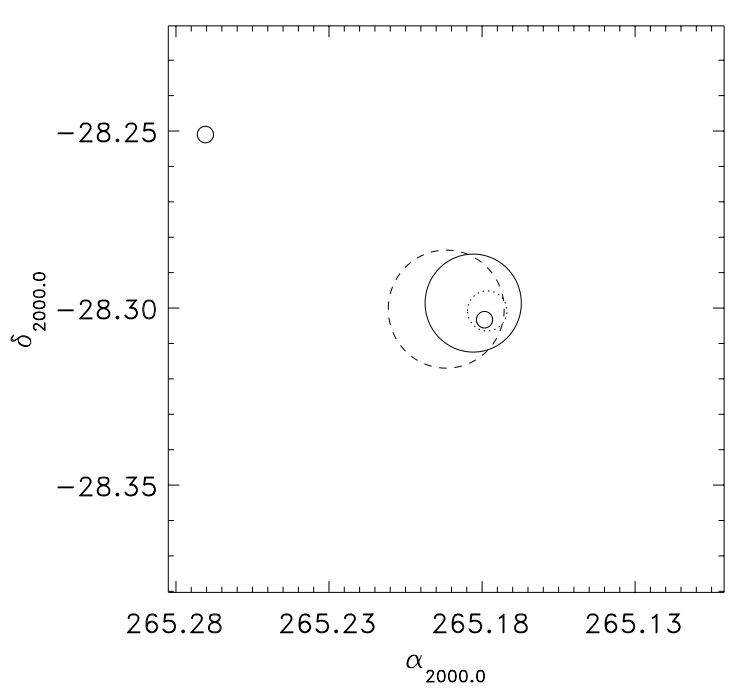

Fig. 1. Locations of Einstein source (large dashed circle), WFC-detected flare (large solid circle), RX J1740.7-2818 (old position / dotted circle, and refined / small solid circle in middle), 2RXP J174104.3-281504 (small solid circle in upper left corner). All circles are at least $90 \%$ confidence.

The event is characterized by a fast rise - within one second - and a factor-of-3.5 decay in the following $880 \mathrm{~s}$ (or an e-folding decay time of $600 \mathrm{~s}$ ). At that point a data gap starts which lasts $1122 \mathrm{~s}$ after which SLX $1737-282$ is not detected anymore. Thus, the flare is shorter than $2000 \mathrm{~s}$.

We modeled the spectrum of the flare successfully with pure black body radiation, see Fig. 2 . This, and the softening of the spectrum during the decay unambiguously identify this flare as a so-called type-I X-ray burst originating from a thermonuclear flash on the surface of a neutron star. The bolometric fluence of the burst before the data gap is $3.0 \times 10^{-5} \mathrm{erg} \mathrm{cm}^{-2}$.

The burst lasts fairly long, with a 2-min long radius expansion phase at the start and a 6.5 min period after that when the flux and temperature retain their maximum values. The radius expansion during the burst suggests that the flux reaches near-Eddington values. This allows a rough estimate of the distance. For an Eddington limit between 2 and $4 \times 10^{38} \mathrm{erg} \mathrm{s}^{-1}$ (Kuulkers et al., in prep.), the peak flux of $(6.0 \pm 0.5) \times 10^{-8} \mathrm{erg} \mathrm{cm}^{-2} \mathrm{~s}^{-1}$ yields a distance between 5 and $8 \mathrm{kpc}$.

\section{BeppoSAX-NFI observations}

A follow-up observation of the burst was carried out with the BeppoSAX Narrow Field Instruments (NFI) half a year later, from 14 October 2000 01:44 UT to 15 October 2000 10:25 UT. Three NFI were turned on: the low-energy concentrator spectrometer LECS (0.1$4 \mathrm{keV}$; Parmar et al. 1997), the medium-energy concentrator spectrometer MECS (1.8-10.5 keV; Boella et al. 1997b), and the Phoswich Detector System PDS (Frontera et al. 1997). The PDS is a collimating instrument with a hexagonal field of view of diameter $1^{\circ} 3$ to 1.5 ; the LECS and MECS are imaging devices with field of views of $40^{\prime}$

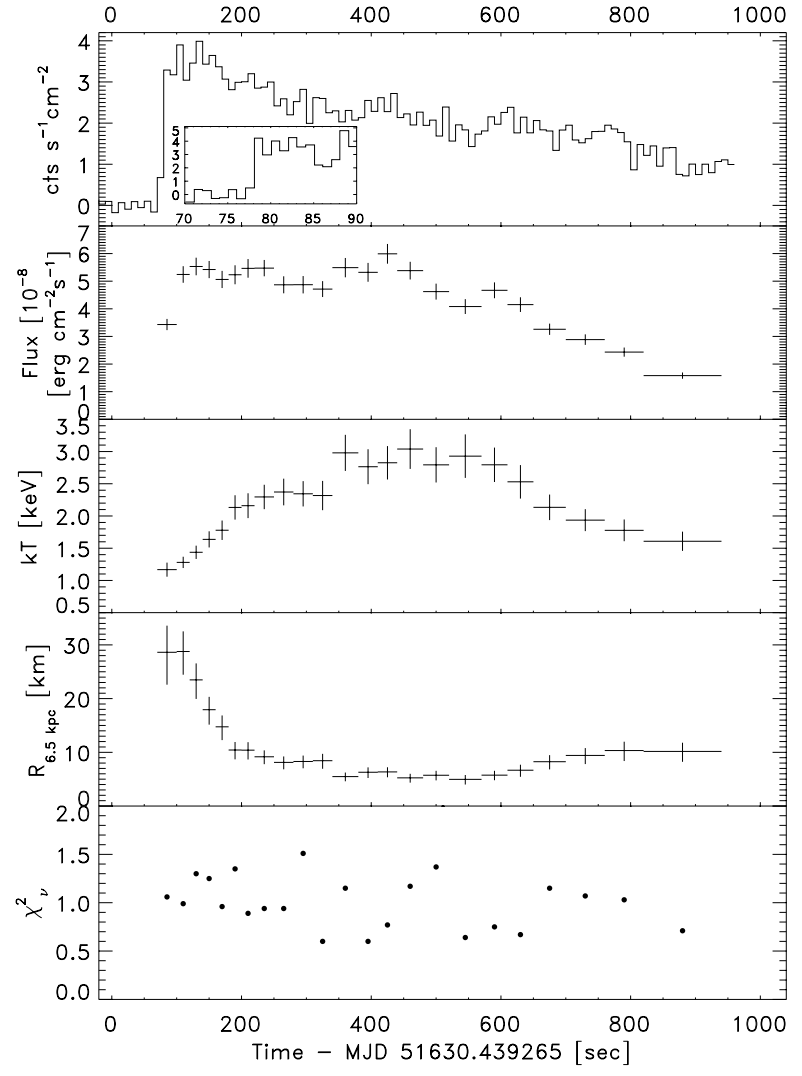

Fig. 2. Time history of WFC-measured full-bandpass photon flux for the flare at $10 \mathrm{~s}$ resolution (top panel; the inset zooms in on the flare onset at $1 \mathrm{~s}$ resolution), and of 4 parameters resulting from modeling with black body radiation keeping $N_{\mathrm{H}}$ fixed at $1.9 \times 10^{22} \mathrm{~cm}^{-2}$ (see Sect. 3): bolometric flux (2nd panel), black body temperature (3rd panel), black body spherical radius for an assumed distance of $6.5 \mathrm{kpc}$ (4th panel), and reduced $\chi^{2}$ value (5th panel).

and $30^{\prime}$ diameter respectively and few arcmin resolution. The exposure times were $14.0 \mathrm{ksec}$ (LECS), $48.0 \mathrm{ksec}$ (MECS) and $22.2 \mathrm{ksec}$ (PDS).

SLX 1737-282 was clearly detected in the LECS and MECS data. Figure 3 shows the light curve as derived from the MECS data. The source exhibits variability with a maximum to minimum ratio of 10 to 1 on typical time scales of a few minutes. The power spectrum shows red noise (i.e., a power-law function with an index of $-1.17 \pm$ 0.04) that becomes indistinguishable from Poisson noise beyond $1 \mathrm{~Hz}$. There are no narrow features. The fractional rms is $40 \pm 1 \%$ between 0.001 and $1 \mathrm{~Hz}$.

A spectrum was generated by combining all LECS, MECS and PDS data. For the data extraction, standard recipes were employed. The off-source pointings of the rocking collimators of the PDS, employed to determine background levels, were verified in WFC data to have no (bright) sources in their field of views. The resulting spectrum could not be modeled in an acceptable way, the reason being that too much flux appears to be contained in the PDS data: all models mandate an unacceptably strong step of a factor of $2.5 \pm 0.1$ between 


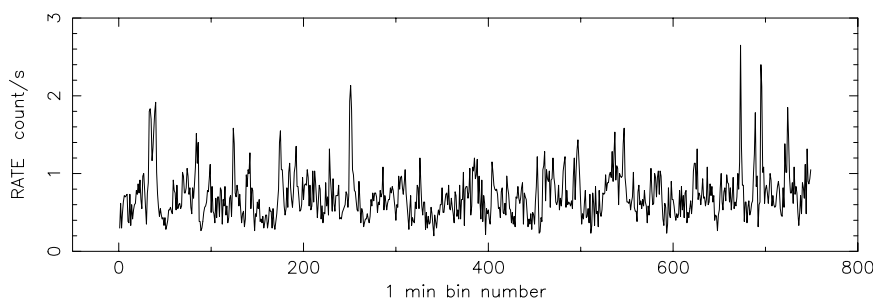

Fig. 3. Time history of photon rate in the MECS with a resolution of 1 min. Twenty one data gaps have been cut out. No background (estimated at $0.02 \mathrm{~s}^{-1}$ ) was subtracted. The typical $1 \sigma$ error per data point is $0.1 \mathrm{c} \mathrm{s}^{-1}$.

the MECS and PDS data. We suspect that either the PDS picks up stray light from the bright source GX $3+1$ that is located 2.36 from SLX 1737-282, or that other faint sources are present inside the field of view of the PDS that confuse the signal from SLX 1737-282 although there are no other sources detected in the (smaller) field of views of LECS and MECS. We decided to exclude the PDS data. The LECS and MECS data can be satisfactorily fit with an absorbed power law $\left(\chi_{\nu}^{2}=1.4\right.$ for $\nu=77$ and a systematic error of $3 \%)$ with a photon index of $2.12 \pm 0.04$ and $N_{\mathrm{H}}=(1.9 \pm 0.1) \times 10^{22} \mathrm{~cm}^{-2}$. These numbers are consistent with the ASCA ones. The observed flux is $6.3 \times 10^{-11} \mathrm{erg} \mathrm{cm}^{-2} \mathrm{~s}^{-1}$ in $0.5-10 \mathrm{keV}$ $\left(1.4 \times 10^{-10} \mathrm{erg}^{-2} \mathrm{sm}^{-1}\right.$ unabsorbed). The PDS data show that the $10-200 \mathrm{keV}$ flux must be below $9.0 \times$ $10^{-11} \mathrm{erg} \mathrm{cm}^{-2} \mathrm{~s}^{-1}$, so that the unabsorbed $0.5-200 \mathrm{flux}$ must be $(1.4-2.3) \times 10^{-10}$ erg $\mathrm{cm}^{-2} \mathrm{~s}^{-1}$.

\section{ROSAT-PSPC observations}

ROSAT carried out a number of pointings on 29 February and 1 March 1992 which cover SLX 1737-282. All of these were with the Position-Sensitive Proportional Counter (PSPC) in 0.5 to $2.4 \mathrm{keV}$. We analyzed these data because they represent the X-ray data with the highest angular resolution and enable a check on source confusion. In the two observations where SLX 1737-282 is almost on axis (obs ids rp400199 and rp400200), we identify two sources close to SLX 1737-282: 1RXS J174043.1281806 (=RX J1740.7-2818) and 2RXP J174104.3-281504. Only the former is consistent with the Einstein position of SLX 1737-282, see Fig. 1, and we identify it with SLX 1737-282. The other is close to Tycho-2 star 6839-218-1, whose colors are compatible with a G star at about $150 \mathrm{pc}$. The detected X-ray flux is compatible with such a counterpart. Using this object to determine the bore sight, and combining both ROSAT observations, we obtain a position for 1RXS J174043.1-281806 of $\alpha_{2000.0}=17^{\mathrm{h}} 40^{\mathrm{m}} 43 \mathrm{~S} .00, \delta_{2000.0}=-28^{\mathrm{o}} 18^{\prime} 11^{\prime \prime} .9$ or $l^{\mathrm{II}}=$ $359.9731, b^{\mathrm{II}}=+1.2481$. The positional accuracy is limited by the statistical error of 2RXP J174104.3-281504 to $8^{\prime \prime} .3$ at $90 \%$ confidence. This is a considerable improvement over the previously determined radius of $20^{\prime \prime} .3$. Motch et al. (1998) attempted to find the optical counterpart of 1RXS J174043.1-281806 in the $I$-band image of this heavily reddened field but were not successful. Within our re-

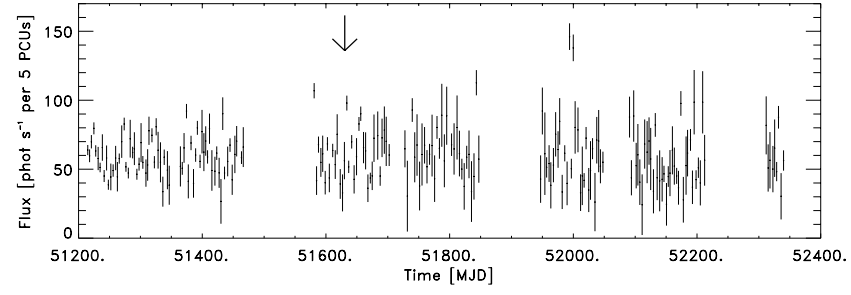

Fig. 4. Time history of the $2-13 \mathrm{keV}$ photon rate of SLX 1737-282 during 206 RXTE-PCA bulge scans, from Feb. 1999 to Mar. 2002. One Crab unit is $10.4 \mathrm{kcts} \mathrm{s}^{-1}$ counted over all 5 proportional counter units (PCUs) that make up the PCA. The arrow indicates the time of the WFC-detected burst.

fined position with 8 ". 3 radius, there are still 11 stars in this image without an obvious candidate (as identified, for instance, by Balmer line emission). We note that the 2MASS catalog shows a bright object within the error circle, but it is too bright ( $K$-dereddened 5.65$)$ to be consistent with SLX 1737-282. The 0.5-2.4 keV spectrum is consistent with the ASCA result. The best-fit $N_{\mathrm{H}}$ for an absorbed power-law with $\Gamma=2.17$ is $(2.2 \pm 0.1) \times$ $10^{22} \mathrm{~cm}^{-2}$ with a flux of $7.2 \times 10^{-12} \mathrm{erg} \mathrm{cm}^{-2} \mathrm{~s}^{-1}$.

\section{RXTE-PCA observations}

Since February 1999, the Proportional Counter Array (PCA) on RXTE has performed scans to monitor a $16^{\circ} \times 16^{\circ}$ region around the Galactic center. Scans occur twice weekly, for a total exposure of approximately 20 seconds per source per observation, and allows detection of source flux variations at the $\sim 1$ mCrab level (Swank \& Markwardt 2001). The temporal coverage is uniform, except for periods from November-January and in June, when the positions of the Sun and anti-Sun cross the galactic center region. Light curves are derived for each known point source in the region. Figure 4 shows that for SLX 1737-282. It shows a fairly steady source with at most a factor of four variability. The mean flux of (60.3 \pm $0.6) \mathrm{c} \mathrm{s}^{-1}$ per 5 PCUs translates, for a Crab spectrum like applies to SLX $1737-282$, to $1.2 \times 10^{-10} \mathrm{erg} \mathrm{cm}^{-2} \mathrm{~s}^{-1}$ in

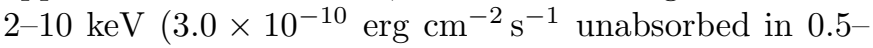
$10 \mathrm{keV})$. However, we note that there is considerable diffuse galactic emission near the galactic center, which can bias the point source light curves rates upwards by several tens of $\mathrm{c} \mathrm{s}^{-1}$ per 5 PCUs. Still, the data show no strong brightening of SLX 1737-282 in 206 measurements.

\section{Discussion}

The detection of the type-I burst from SLX 1737-282 unambiguously identifies the origin as a neutron star. By analogy with other type-I X-ray bursters, it is almost certain that this neutron star is in a low-mass X-ray binary (LMXB) with a sub-solar mass companion. According to a count based on the LMXB catalog by Liu et al. (2001) which was updated with recent discoveries, SLX 1737-282 is the 69th X-ray burster (cf., In 't Zand 2001). Among a LMXB population of about 150, this means that at least 
Table 1. History of X-ray flux from SLX 1737-282.

\begin{tabular}{lllll}
\hline \hline Data set & Time & $\begin{array}{l}\text { Band }^{*} \\
(\mathrm{keV})\end{array}$ & Flux $^{\dagger}$ & $\begin{array}{l}\text { Flux }^{\ddagger} \text { in } \\
0.5-10 \mathrm{keV}\end{array}$ \\
\hline SL2-XRT & Jul 85 & $3-30$ & 7.7 & $13 \pm 3$ \\
ROSAT-PSPC & Feb 92 & $0.5-2.4$ & 0.72 & $10 \pm 1$ \\
ASCA-GIS & Sep 96 & $0.7-10$ & 5.7 & $13 \pm 3$ \\
ASCA-GIS & Sep 98 & $0.7-10$ & 6.5 & $14 \pm 3$ \\
ASCA-GIS & Mar 99 & $0.7-10$ & 4.8 & $11 \pm 2$ \\
BSAX-NFI & Oct 00 & $0.5-10$ & 6.3 & $14 \pm 3$ \\
\hline
\end{tabular}

${ }^{*}$ Bandpass in which flux originally was measured; ${ }^{\dagger}$ Original flux measurement in $10^{-11} \mathrm{erg} \mathrm{cm} \mathrm{cm}^{-2} \mathrm{~s}^{-1}$; ${ }^{\ddagger}$ Flux in $0.5-10 \mathrm{keV}$ band, as extrapolated by assuming power law with photon index $\Gamma=2.15$ and $N_{\mathrm{H}}=1.9 \times 10^{22} \mathrm{~cm}^{-2}$. The errors are propagated errors in $\Gamma$ of 0.1 and in $N_{\mathrm{H}}$ of $0.1 \times 10^{22} \mathrm{~cm}^{-2}$.

about half of all LMXBs contain neutron stars. Identifying the companion star in the optical may prove difficult, because of its proximity to the galactic center and the high reddening $\left(A_{\mathrm{V}} \approx 11\right)$ implied by the value of $N_{\mathrm{H}}$.

Whenever the average $0.5-10 \mathrm{keV}$ flux of SLX 1737-282 is measured with sufficient accuracy (see Table 1 ) it is in a narrow range of 1.0 to $1.4 \times 10^{-10}$ erg $\mathrm{cm}^{-2} \mathrm{~s}^{-1}$. The NFI observation shows that this may extrapolate to as much as $2.3 \times 10^{-10} \mathrm{erg} \mathrm{cm}^{-2} \mathrm{~s}^{-1}$ in $0.5-200 \mathrm{keV}$. The RXTE data show that during several hundred brief monitoring observations in 3 years the source never shows signs of strong brightening. Given the distance, the $0.5-200 \mathrm{keV}$ flux translates to a luminosity of $5 \times 10^{35}$ to $1.8 \times 10^{36} \mathrm{erg} \mathrm{s}^{-1}$, suggesting a bolometric luminosity that is less than or similar to $1 \%$ of the Eddington limit (as is also suggested by a direct comparison between the peak flux of the Eddington-limited burst and the persistent flux).

If one ignores "superbursts" (e.g., Kuulkers et al. 2002a), the burst duration and fluence are among the largest (cf., Tawara et al. 1984; Lewin et al. 1984; Van Paradijs et al. 1990; Swank et al. 1977; Kaptein et al. 2000; Kuulkers et al. 2002b). The amount of radiated energy, if isotropic, is between 1.0 and $2.2 \times 10^{41} \mathrm{erg}$. If the energy released per gram of accreted material is between 1.6 and $6 \times 10^{18} \mathrm{erg}$, this is equivalent to $2 \times 10^{22}$ to $1.4 \times 10^{23} \mathrm{~g}$ of accreted material. Given the persistent luminosity of perhaps a few times $10^{36}$ erg $\mathrm{s}^{-1}$, the mass accretion rate may be 5 to $10 \times 10^{15} \mathrm{~g} \mathrm{~s}^{-1}$ (or 1 to $2 \times 10^{-10} M_{\odot} \mathrm{yr}^{-1}$ ). This implies a waiting time between bursts between 20 and 320 days, consistent with the detection of just a single burst. The long duration combined with the low persistent flux suggests that the burst is caused by a thermonuclear flash in a hydrogen-rich environment in a mass accretion regime of $\dot{M}<2 \times 10^{-10} M_{\odot} \mathrm{yr}^{-1}$ (Fujimoto et al. 1981).

There is a slight similarity to sources like 2S 0918-549 (e.g., Jonker et al. 2001) and 4U 1850-087 (e.g., Kitamoto et al. 1992). These are also rare bursters and moderately bright sources that, foremost, show broad emission features at about $0.7 \mathrm{keV}$ attributable to an overabundance of Ne which would be consistent with a hydrogen-depleted donor in an ultracompact orbit (Juett et al. 2001). Our data are not constraining with regards to the $0.7 \mathrm{keV}$ feature due to the high value of $N_{\mathrm{H}}$, but the hydrogen-rich environment inferred from the burst duration suggests that the similarity is superficial. In this respect we note that the burst in SLX 1737-282 is an order of magnitude longer than in 2S 0918-549 and 4U 1850-087.

Acknowledgements. JZ and EK acknowledge financial support from the Netherlands Org. for Scientific Research (NWO).

\section{References}

Boella, G., Butler, R., Perola, G., et al. 1997a, A\&AS, 122, 299

Boella, G., Chiappetti, L., Conti, G., et al. 1997b, A\&AS, 122, 327

Emelyanov, A. N., Aleksandrovich, N. L., \& Sunyaev, R. A. 2000, Astr. Lett., 26, 297

Frontera, F., Costa, E., Dal Fiume, D., et al. 1997, A\&AS, 122, 357

Fujimoto, M. Y., Hanawa, T., \& Miyaji, S. 1981, ApJ, 247, 267

Hertz, P., \& Grindlay, J. E. 1983, ApJ, 275, 105

In 't Zand, J. J. M. 1992, Ph.D. Thesis, University of Utrecht

In 't Zand, J. J. M. 2001, in Proc. 4th INTEGRAL workshop (Sep. 2000, Alicante, Spain), ed. A. Giminez, V. Reglero, \& C. Winkler, 463

Jager, R., Mels, W., Brinkman, A., et al. 1997, A\&AS, 125, 557

Jonker, P. G., van der Klis, M., Homan, J., et al. 2001, ApJ, 553,335

Juett, A. M., Psaltis, D., \& Chakrabarty, D. 2001, ApJ, 534, 380

Kaptein, R., In 't Zand, J. J. M., Kuulkers, E., et al. 2000, A\&A, 358, L71

Kitamoto, S., Tsunemi, H., \& Roussel-Dupre, D. 1992, ApJ, 391, 220

Kuulkers, E., in 't Zand, J. J. M., Van Kerkwijk, M. H., et al. 2002a, A\&A, 382, 503

Kuulkers, E., Homan, J., Van der Klis, M., Lewin, W. H. G., \& Mendez, M. 2002b, A\&A, 382, 947

Lewin, W. H. G., Vacca, W., \& Basinska, E. 1984, ApJ, 277, L57

Liu, Q. Z., van Paradijs, J., \& van den Heuvel, E. P. J. 2001, A\&A, 368, 1021

Motch, C., Guillout, P., Haberl, F., et al. 1998, A\&AS, 132, 341

Parmar, A. N., Martins, D. D. E., Bavdaz, M., et al. 1997, A\&AS, 122, 309

Pavlinsky, M. N., Grebenev, S. A., \& Runyaev, R. A. 1994, ApJ, 425, 110

Sakano, M., Koyama, K., Murakami, H., Maeda, Y., \& Yamauchi, S. 2002, ApJS, 138, 19

Skinner, G. K., Willmore, A. P., \& Eyles, C. J. 1987, Nature, 330,544

Swank, J., Becker, R. H., Boldt, E. A., et al. 1977, ApJ, 212, L73

Swank, J., \& Markwardt, C. 2001, in Proc. New Century of X-ray Astronomy, ed. H. Kunieda, \& H. Inoue, PASP Conf. Ser., in press

Tawara, Y., Kii, T., Hayakawa, S., et al. 1984, ApJ, 276, L41

Van Paradijs, J., Dotani, T., Tanaka, Y., \& Tsuru, T. 1990, PASJ, 42, 633 